\title{
ON THE LAW OF THE ITERATED LOGARITHM FOR UNIFORMLY BOUNDED ORTHONORMAL SYSTEMS
}

\author{
BY \\ MARY WEISS
}

It is a familiar fact that certain systems of functions, although themselves not independent, exhibit properties of independent random variables. For example it is well known that if $\left\{n_{k}\right\}$ is a lacunary sequence of positive integers, that is $n_{k+1} / n_{k}>q>1$, then the sequence

$$
\left\{\rho_{k} \cos \left(n_{k}+\alpha_{k}\right)\right\}
$$

where the $\alpha_{k}$ are arbitrary and the $\rho_{k}$ satisfy certain order of magnitude conditions, obeys both the Central Limit Theorem and the Law of the Iterated Logarithm( ${ }^{1}$. The system (1) is obtained by taking a sparse subsequence of the trigonometric system, and it is natural to ask whether in every uniformly bounded orthonormal system it is possible to select a subsystem imitating independent random variables. In this direction, Morganthaler [6] has obtained the following analogue of the Central Limit Theorem.

THEOREM A. Let $\left\{\phi_{n}(x)\right\}$ be a uniformly bounded orthonormal system of real-valued functions on the interval $[0,1]$. Then there exists a subsequence $\left\{\phi_{n_{k}}(x)\right\}$ and a real-valued function $f(x), \int_{0}^{1} f^{2}(x) d x=1,0 \leqq f(x) \leqq B$ where $B$ is the uniform bound of $\left\{\phi_{n}(x)\right\}$, such that for any arbitrary sequence $\left\{a_{k}\right\}$ of real numbers satisfying

$$
\begin{aligned}
A_{N} & =\left(a_{1}^{2}+a_{2}^{2}+\cdots+a_{N}^{2}\right)^{1 / 2} \rightarrow \infty \text { as } N \rightarrow \infty, \\
a_{N} & =o\left(A_{N}\right),
\end{aligned}
$$

the distribution functions

$$
F_{N}(y)=\left|\left\{x: \sum_{k=1}^{N} a_{k} \phi_{n_{k}}(x) / A_{N} \leqq y\right\}\right|
$$

converge to a limiting distribution $F(y)$ at each point of continuity of the latter and the characteristic function of $F(y)$ is

$$
\Phi(\lambda)=\int_{0}^{1} \exp \left(-\lambda^{2} f^{2}(x) / 2\right) d x .
$$

It will be seen from (2) that the limiting distribution $F(y)$ is not a true Gaussian, but is a sort of Gaussian distribution with a weight function $f(x)$.

Presented to the Society, February 25, 1956; received by the editors June 11, 1958.

(1) In this connection see references 1-6 at the end of paper. 
The purpose of this paper is to prove an analogue of the Law of the Iterated Logarithm for orthonormal systems. We have the following theorem.

THEOREM 1. Let $\left\{\phi_{n}(x)\right\}$ be a uniformly bounded orthonormal system of realvalued functions on the interval $[0,1]$. Then there exists a subsequence $\left\{\phi_{n_{k}}(x)\right\}$ and a real-valued function $f(x), \int_{0}^{1} f^{2}(x) d x=1,0 \leqq f(x) \leqq B$, where $B$ is the uniform bound of $\left\{\phi_{n}(x)\right\}$, such that for any arbitrary sequence $\left\{a_{k}\right\}$ of real num. bers satisfying

$$
\begin{aligned}
& A_{N}=\left(a_{1}^{2}+a_{2}^{2}+\cdots+a_{N}^{2}\right)^{1 / 2} \rightarrow \infty \quad \text { as } N \rightarrow \infty, \\
& M_{N}=o\left(A_{N}\left(\log \log A_{N}\right)^{-1 / 2}\right) \quad \text { where } M_{N}=\max _{k \leqq N}\left|a_{k}\right|
\end{aligned}
$$

we have

$$
\lim \sup \frac{S_{N}(x)}{\left(2 A_{N}^{2} \log \log A_{N}\right)^{1 / 2}}=f(x)
$$

where

$$
S_{N}(x)=\sum_{k=1}^{N} a_{k} \phi_{n_{k}}(x)
$$

We note that conditions (3) are the same as the conditions on the deviations and maxima of the independent random variables in the theorem of Kolmogoroff [7] on the Law of the Iterated Logarithm, and that the conclusion there is the same as (4) except that for independent random variables $f(x)$ is equal to 1 . In Theorem 1 the function $f(x)$ can be taken to be the same as in Theorem A.

We begin by extracting a subsequence $\left\{\phi_{n_{k}}(x)\right\}$ of $\left\{\phi_{n}(x)\right\}$ for which there is a function $f(x)$ such that

$$
\phi_{n_{k}}^{2} \underset{\text { weakly }}{\longrightarrow} f^{2}(x)
$$

that is to say, $f(x)$ has the property that for every integrable function $g(x)$,

$$
\int_{0}^{1} g(x) \phi_{n_{k}}^{2}(x) d x \rightarrow \int_{0}^{1} g(x) f^{2}(x) d x .
$$

It is well known that the only condition needed on the sequence $\left\{\phi_{n}\right\}$ in order to find such a weakly convergent subsequence is that it be uniformly bounded. From now on we will denote the subsequence $\left\{\phi_{n_{k}}\right\}$ so chosen by $\left\{\phi_{n}\right\}$.

Now the method of selection of the subsequence of Theorem 1 will involve, for values of $x$ for which $f(x)>0$, the use of $\phi_{n_{k}}(x) / f(x)$ and sums, products 
and integrals of such expressions. If $f(x)$ is not bounded away from zero then $\phi_{n_{k}}(x) / f(x)$ may not be integrable. Therefore we first prove our theorem for a fixed arbitrary set $F$ on which $f(x)$ is bounded from zero and for the set $E$ on which $f(x)$ is zero. Then by using a diagonalizing procedure we can obtain a subsequence which satisfies (4) for almost every $x$. That is, we will prove the following theorem which implies Theorem 1.

THEOREM 1'. If $\left\{\phi_{n}(x)\right\}$ is a sequence of functions satisfying the hypotheses of Theorem 1 for which the $\phi_{n}^{2}(x)$ converge weakly to a function $f^{2}(x)$, and if $F$ is an arbitrary fixed set on which $f(x)$ is bounded away from zero, and $E$ is the set on which $f(x)$ is equal to zero then there exists a subsequence $\left\{\phi_{n_{k}}(x)\right\}$ such that for any sequence $\left\{a_{k}\right\}$ satisfying the hypotheses of Theorem 1 we have

$$
\lim \sup \frac{S_{N}(x)}{\left(2 A_{N}^{2} \log \log A_{N}\right)^{1 / 2}}=f(x)
$$

almost everywhere in $E \cup F$.

There are two main properties of our uniformly bounded orthonormal system which we will use repeatedly in the proof which follows. The first is the fact that the $\phi_{n}^{2}(x)$ converge weakly to $f^{2}(x)$. The second is the fact that for any integrable $g(x)$,

$$
\int_{0}^{1} g(x) \phi_{n}(x) d x \underset{n \rightarrow \infty}{\longrightarrow} 0 .
$$

This is the well-known Riemann-Lebesgue theorem and it holds for any uniformly bounded orthonormal system.

We will divide the proof of Theorem 1' into two parts.

Part I. This part of the paper will be devoted to choosing a subsequence for which

$$
\lim \sup \frac{S_{N}(x)}{\left(2 A_{N}^{2} \log \log A_{N}\right)^{1 / 2}} \leqq f(x) .
$$

We first prove three lemmas.

Lemma 1. Let $F$ be a fixed set on which $f(x)$ is bounded away from zero. Then we can choose a subsequence $\left\{\phi_{n_{k}}\right\}$ of $\left\{\phi_{n}\right\}$ for which

$$
\begin{aligned}
\frac{1}{2}|F| \exp \left(\frac{\lambda^{2} A_{N}^{2}}{2}(1-\eta)\right) & \leqq \int_{F} \exp \left(\frac{\lambda S_{N}(x)}{f(x)}\right) d x \\
& \leqq \frac{3}{2}|F| \exp \left(\frac{\lambda^{2} A_{N}{ }^{2}}{2}(1+\eta)\right)
\end{aligned}
$$


where $S_{N}, A_{N}$, and $M_{N}$, have the same meaning as in the theorem and $\lambda, \eta$ are any positive numbers satisfying

$$
2 C \alpha^{3} \lambda M_{N}+\left(\lambda M_{N}\right)^{2} / 2<\eta, \quad \lambda M_{N} \alpha<1 / 2
$$

where $\alpha=\max _{x, n}\left|\phi_{n}(x) / f(x)\right|$ and $C$ is any constant for which inequality (10), below, holds.

The proof is based on the inequality

$$
\left|\log \left(1+z+z^{2} / 2\right)-z\right| \leqq C|z|^{3},
$$

valid for $|z| \leqq 1 / 2$. From the second of the conditions (9), we see that we may take $z=\lambda a_{k} \phi_{n_{k}} / f(x)$. We obtain

$$
\exp \left(\frac{\lambda a_{k} \phi_{n_{k}}}{f(x)}\right)=\left(1+\frac{\lambda a_{k} \phi_{n_{k}}}{f(x)}+\left(\frac{\lambda a_{k} \phi_{n_{k}}}{f(x)}\right)^{2} \cdot \frac{1}{2}\right) e^{R_{k}(x)}
$$

where

$$
\left|R_{k}\right| \leqq C\left|\lambda a_{k} \phi_{n_{k}} / f(x)\right|^{3} \leqq C \lambda{ }^{3} a_{k}^{2} M \alpha^{3} .
$$

Clearly (11) and (12) imply

$$
\begin{aligned}
\exp \left(-C \lambda M \alpha^{3} \lambda^{2} A_{N}^{2}\right) & \int_{F} \prod_{1}^{N}\left(1+\frac{\lambda a_{k} \phi_{n_{k}}}{f(x)}+\frac{1}{2}\left(\frac{\lambda a_{k} \phi_{n_{k}}}{f(x)}\right)^{2}\right) d x \\
& \leqq \int_{F} \exp \left(\frac{\lambda S_{N}(x)}{f(x)}\right) d x \\
& \leqq \exp \left(C \lambda M \alpha^{3} \lambda^{2} A_{N}^{2}\right) \int_{F} \prod_{1}^{N}\left(1+\frac{\lambda a_{k} \phi_{n_{k}}}{f(x)}+\frac{1}{2}\left(\frac{\lambda a_{k} \phi_{n_{k}}}{f(x)}\right)^{2}\right) d x
\end{aligned}
$$

Inequalities (13) hold no matter what the subsequence $\left\{\phi_{n_{k}}\right\}$. Next we pick $\left\{\phi_{n_{k}}\right\}$. We do so by induction. In order to simplify notation we write $\psi_{n}$ $=\phi_{n} / f(x)$. First we fix two series of positive numbers, $\sum_{1}^{\infty} \epsilon_{k}$ and $\sum_{1}^{\infty} \epsilon_{k}^{\prime}$, satisfying $\sum_{1}^{\infty} \epsilon_{k}^{\prime}+\sum_{1}^{\infty} k \epsilon_{k} \leqq 1 / 2$. Then we choose $\phi_{n_{k}}$ inductively to satisfy

$$
\begin{aligned}
& \sum_{\left(\beta_{1}, \beta_{2}, \cdots, \beta_{k-1}\right)}\left|\int_{F} \psi_{n_{1}}^{\beta_{1}} \cdots \psi_{n_{k-1}}^{\beta_{k-1}} \psi_{n_{k}} d x\right| \leqq \epsilon_{k}^{\prime}|F|, \\
& \sum_{\left(\beta_{1}, \beta_{2}, \cdots, \beta_{k-1}\right)}\left|\int_{F} \psi_{n_{1}}^{\beta_{1}} \cdots \psi_{n_{k-1}}^{\beta_{k-1}} \psi_{n_{k}}^{2}-\psi_{n_{1}}^{\beta_{1}} \cdots \psi_{n_{k-1}}^{\beta_{k-1}} d x\right| \leqq \epsilon_{k}|F|
\end{aligned}
$$

where the sums are taken over all possible $k-1$ tuples with elements in $\{0,1,2\}$. It is clear that the Riemann Lebesgue theorem and the weak convergence principle allow us to do this.

Now we estimate 


$$
\begin{aligned}
\mathfrak{I}= & \int_{F} \prod_{1}^{N}\left(1+\lambda a_{k} \psi_{n_{k}}+\frac{1}{2}\left(\lambda a_{k} \psi_{n_{k}}\right)^{2}\right) d x=\int_{F} \prod_{1}^{N}\left(1+\frac{1}{2}\left(\lambda a_{k} \psi_{n_{k}}\right)^{2}\right) d x \\
& +\int_{F}\left[\prod_{1}^{N}\left(1+\lambda a_{k} \psi_{n_{k}}+\frac{1}{2}\left(\lambda a_{k} \psi_{n_{k}}\right)^{2}\right)-\prod_{1}^{N}\left(1+\frac{1}{2}\left(\lambda a_{k} \psi_{n_{k}}\right)^{2}\right)\right] d x \\
= & P+Q .
\end{aligned}
$$

We first investigate $Q$. We note that it is the sum of those terms in the expansion of $\mathfrak{g}$ which are integrals of products containing at least one $\psi_{n_{k}}$ raised to the power 1 . Such a term is of the form

$$
\begin{aligned}
\frac{\left(\lambda a_{1}\right)^{\beta_{1}}}{2_{1}^{\beta^{\prime}}} \cdots \frac{\left(\lambda a_{k-1}\right)^{\beta k-1}}{2_{k-1}^{\beta^{\prime}}} \lambda a_{k} \frac{\left(\lambda a_{j_{1}}\right)^{2}}{2} & \cdots \frac{\left(\lambda a_{j_{g}}\right)^{2}}{2} \\
& \cdot \int_{F} \psi_{n_{1}}^{\beta_{1}} \cdots \psi_{n_{k-1}}^{\beta_{k-1}} \psi_{n_{k}} \psi_{n_{j_{1}}}^{2} \cdots \psi_{n_{j_{j}}}^{2} d x
\end{aligned}
$$

where $\psi_{n_{k}}$ is the function with the highest index which is raised to the power $1, \beta_{i}^{\prime}=1$ if $\beta_{i}=2, \beta_{i}^{\prime}=0$ if $\beta_{i}=1$ or $\beta_{i}=0$. Since $\lambda a_{j}<1 / 2$, the absolute value of such a term is less than

$$
\frac{\left(\lambda a_{j_{1}}\right)^{2}}{2} \cdots \frac{\left(\lambda a_{j_{g}}\right)^{2}}{2}\left|\int_{F} \psi_{n_{1}}^{\beta_{1}} \cdots \psi_{n_{k-1}}^{\beta_{k-1}} \psi_{n_{k}} \psi_{n_{j_{1}}}^{2} \psi_{n_{j_{2}}}^{2} \cdots \psi_{n_{j_{s}}}^{2} d x\right| .
$$

We fix $n_{k}$ and $\left(n_{j_{1}}, \cdots, n_{j_{s}}\right)$, and consider the sum over all $\left(\beta_{1}, \cdots, \beta_{k-1}\right)$ of terms of type (16). We call this sum $G\left(n_{k}, n_{j_{1}}, \cdots, n_{j_{s}}\right)$. It is clear from (14) and (15) that

$$
\begin{aligned}
& G\left(n_{k}, n_{j_{1}}, \cdots, n_{j_{s}}\right) \leqq \frac{\left(\lambda a_{j_{1}}\right)^{2}}{2} \cdots \frac{\left(\lambda a_{j_{g}}\right)^{2}}{2} \sum_{\left(\beta_{1}, \cdots, \beta_{k-1}\right)}\left|\int_{F} \psi_{n_{1}}^{\beta_{1}} \cdots \psi_{n_{k-1}}^{\beta_{k-1}} \psi_{n_{k}} d x\right| \\
& +\frac{\left(\lambda a_{j_{1}}\right)^{2}}{2} \cdots \frac{\left(\lambda a_{j_{s}}\right)^{2}}{2}|F| \sum_{j>k} \epsilon_{j} \leqq \frac{\left(\lambda a_{j_{1}}\right)^{2}}{2} \cdots \frac{\left(\lambda a_{j_{s}}\right)^{2}}{2}|F|\left(\epsilon_{k}^{\prime}+\sum_{j>k} \epsilon_{j}\right) .
\end{aligned}
$$

Hence

$$
\begin{aligned}
|Q| & \leqq \sum_{j_{1}<j_{2}<\cdots<j_{0}<N} \sum_{n_{k} \leq N} G\left(n_{k}, n_{j_{1}}, \cdots, n_{j_{0}}\right) \\
& \leqq|F|\left(\sum_{1}^{\infty} \epsilon_{k}^{\prime}+\sum_{1}^{\infty} k \epsilon_{k}\right) \sum_{j_{1}<\cdots<j_{o}<N} \frac{\left(\lambda a_{j_{1}}\right)^{2}}{2} \cdots \frac{\left(\lambda a_{j_{0}}\right)^{2}}{2} \\
& \leqq \frac{1}{2}|F| \prod_{1}^{N}\left(1+\frac{\lambda^{2} a_{k}^{2}}{2}\right) .
\end{aligned}
$$

Now we estimate $P$. Clearly, 


$$
P=|F|+\sum_{j_{1}<\cdots<j_{p}<N} \frac{\lambda^{2 p}}{2^{p}} a_{j_{1}}^{2} \cdots a_{j_{p}}^{2} \int_{F} \psi_{n_{j_{1}}}^{2} \cdots \psi_{n_{j_{p}}}^{2} d x .
$$

From (15) it follows that

$$
\left|\int_{F} \psi_{n_{j_{1}}}^{2} \cdots \psi_{n_{j_{p}}}^{2} d x-\right| F|| \leqq|F| \sum_{1}^{N} \epsilon_{k} .
$$

Hence

$$
\begin{aligned}
|F|+|F|(1 & \left.-\sum_{1}^{\infty} \epsilon_{k}\right) \sum_{j_{1}<\cdots<j_{p}<N} \frac{\lambda^{2 p}}{2^{p}} a_{j_{1}}^{2} \cdots a_{j_{p}}^{2} \\
& \leqq P \leqq|F|+|F|\left(1+\sum_{1}^{\infty} \epsilon_{k}\right) \sum_{j_{1}<\cdots<j_{p}<N} \frac{\lambda^{2 p}}{2^{p}} a_{j_{1}}^{2} \cdots a_{j_{p}}^{2}
\end{aligned}
$$

which implies

$$
\begin{aligned}
|F|\left(1-\sum_{1}^{\infty} \epsilon_{k}\right) \prod_{1}^{N}\left(1+\frac{\left(\lambda a_{k}\right)^{2}}{2}\right) & \\
& \leqq P \leqq|F|\left(1+\sum_{1}^{\infty} \epsilon_{k}\right) \prod_{1}^{N}\left(1+\frac{\left(\lambda a_{k}\right)^{2}}{2}\right) .
\end{aligned}
$$

Collecting (18) and (19) we have

$$
\begin{aligned}
\frac{1}{2}|F| \prod_{1}^{N}\left(1+\frac{\lambda^{2} a_{k}^{2}}{2}\right) & \leqq \int_{F} \prod_{1}^{N}\left(1+\lambda a_{k} \psi_{n_{k}}+\frac{1}{2}\left(\lambda a_{k} \psi_{n_{k}}\right)^{2}\right) d x \\
& \leqq \frac{3}{2}|F| \prod_{1}^{N}\left(1+\frac{\left(\lambda a_{k}\right)^{2}}{2}\right) .
\end{aligned}
$$

The inequality $e^{u(1-u)} \leqq 1+u \leqq e^{u}$, valid for positive $u$ implies

$$
\exp \left(\sum_{1}^{N} \frac{\lambda^{2} a_{k}^{2}}{2}-\sum_{1}^{N} \frac{\lambda^{4} a_{k}^{4}}{4}\right) \leqq \prod_{1}^{N}\left(1+\frac{\lambda^{2} a_{k}^{2}}{2}\right) \leqq \exp \left(\sum \frac{\lambda^{2} a_{k}^{2}}{2}\right) .
$$

From (13), (20), and (21) it follows that

$$
\begin{aligned}
\frac{1}{2}|F| \exp ( & \left.\frac{\lambda^{2} A_{N}^{2}}{2}\left(1-\frac{\lambda^{2} M^{2}}{2}-2 C \lambda M \alpha^{8}\right)\right) \\
& \leqq \int_{F} \exp \left(\lambda \frac{S_{N}(x)}{f(x)}\right) d x \leqq \frac{3}{2}|F| \exp \left(\frac{\lambda{ }^{2} A_{N}^{2}}{2}\left(1+2 C \lambda M \alpha^{8}\right)\right) .
\end{aligned}
$$

Applying the first of conditions (9) to this inequality we obtain the conclusion of our lemma. 
Lemma 2. Let $E$ be the set on which $f(x)=0$ and let

$$
\lambda M_{N} \leqq \frac{1}{2}, \quad C \lambda M_{N}<\eta .
$$

Then there exists a subsequence $\left\{\phi_{n_{k}}\right\}$ for which

$$
\int_{E} \exp \left(\lambda S_{N}(x)\right) d x \leqq 2 \exp \left(\eta \lambda^{2} A_{N}^{2}\right) .
$$

We choose $\left\{\phi_{n_{k}}\right\}$ inductively to satisfy

$$
\begin{array}{r}
\sum_{\left(\beta_{1}, \cdots, \beta_{k-1}\right)}\left|\int_{E} \phi_{n_{1}}^{\beta_{1}} \phi_{n_{2}}^{\beta_{2}} \cdots \phi_{n_{k-1}}^{\beta_{k-1}} \phi_{n_{k}} d x\right| \leqq \epsilon_{k}^{\prime}, \\
\sum_{\left(\beta_{1}, \cdots, \beta_{k-1}\right)}\left|\int_{E} \phi_{n_{1}}^{\beta_{1}} \phi_{n_{2}}^{\beta_{2}} \cdots \phi_{n_{k-1}}^{\beta_{k-1}} \phi_{n_{k}}^{2} d x\right| \leqq \epsilon_{k},
\end{array}
$$

where $\boldsymbol{\epsilon}_{k}, \boldsymbol{\epsilon}_{k}^{\prime}$ and $\beta_{i}$ are the same as in Lemma 1. We can choose $\left\{\phi_{n_{k}}\right\}$ to satisfy (22) by the Riemann-Lebesgue and to satisfy (23) because of the fact that on $E$ the weak limit of $\phi_{n}^{2}$ is zero.

Following the proof of Lemma 1 we see that

$$
\int_{E} \exp \left(\lambda S_{N}\right) d x \leqq \exp \left(C \lambda M \frac{\lambda \stackrel{2}{A}_{N}^{2}}{2}\right) \int_{E} \prod_{1}^{N}\left(1+\lambda a_{k} \phi_{n_{k}}+\frac{\left(\lambda a_{k} \phi_{n_{k}}\right)^{2}}{2}\right) d x .
$$

Now we will show that

$$
\int_{E} \prod_{1}^{N}\left(1+\lambda a_{k} \phi_{n_{k}}+\frac{\left(\lambda a_{k} \phi_{n_{k}}\right)^{2}}{2}\right) d x \leqq 2 .
$$

Let us consider all terms in the expansion of the left side of (24) whose nonconstant factor of highest index is $\phi_{n_{k}}$. Since the conditions of the lemma imply $\lambda a_{k}<1 / 2$, (22) implies that the sum of all such terms is less than $\epsilon_{k}^{\prime}$. Similarly (23) implies that the sum of all terms in the expansion of (24) whose nonconstant factor of highest index is $\phi_{n_{k}}^{2}$ is less than $\epsilon_{k}$. Thus

$$
\int \prod_{E}^{N}\left(1+\lambda a_{k} \phi_{n_{k}}+\frac{\left(\lambda a_{k} \phi_{u_{k}}\right)^{2}}{2}\right) d x \leqq 1+\sum_{1}^{N} \epsilon_{k}+\sum_{1}^{N} \epsilon_{k}^{\prime} \leqq 2 .
$$

Hence

$$
\int_{E} e^{\lambda S_{N}} d x \leqq 2 \exp \left(C \lambda M \lambda^{2} A_{N}^{2}\right) \leqq 2 \exp \left(\eta \lambda^{2} A_{N}^{2}\right) .
$$

Lemma 3. We can choose a subsequence $\left\{\phi_{n_{k}}\right\}$ of $\left\{\phi_{n}\right\}$ such that if $\left\{a_{\nu}\right\}$ is any semuence for which $-2^{\nu} \leqq a_{\nu} \leqq 2^{\nu}$ and if 


$$
\begin{aligned}
& e_{i, N, \epsilon}=\left\{x: \begin{array}{l}
S_{i}(x)>f(x)(1+\epsilon)\left(2 A_{N}^{2} \log \log A_{N}\right)^{1 / 2} \text { and } \\
S_{l}(x) \leqq f(x)(1+\epsilon)\left(2 A_{N}^{2} \log \log A_{N}\right)^{1 / 2} \text { for all } l<i
\end{array}\right\}, \\
& e_{i, N, \epsilon}^{\prime}=\left\{x: \begin{array}{l}
x \in E, S_{i}(x)>\epsilon\left(2 A_{N}^{2} \log \log A_{N}\right)^{1 / 2} \text { and } \\
S_{l}(x) \leqq \epsilon\left(2 A_{N}^{2} \log \log A_{N}\right)^{1 / 2} \text { for all } l<i
\end{array}\right\}
\end{aligned}
$$

then

$$
\begin{gathered}
\int_{e_{i, N, \epsilon}}\left(\phi_{n_{j}} \phi_{n_{k}} / f^{2}(x)\right) d x \leqq 4^{-k}\left|e_{i, N, \epsilon}\right| \\
\left.\int_{e i, N, \epsilon}{ }^{2} \phi_{n_{k}}^{2} / f^{2}(x)\right) d x \leqq(1+1 / 4)\left|e_{i, N, \epsilon}\right| \\
\int_{e_{i, N, \epsilon}} \phi_{n_{j}} \phi_{n_{k}} d x \leqq 4^{-k}\left|e_{i, N, \epsilon}^{\prime}\right| \\
\int_{e_{i, N, \epsilon}^{\prime}} \phi_{n_{k}}^{2} d x \leqq(1 / 4)\left|e_{i, N, \epsilon}^{\prime}\right|
\end{gathered}
$$

provided $N<i<j<k,\left|e_{i, N, \epsilon}\right|>1 / k^{2},\left|e_{i, N, \epsilon}^{\prime}\right|>1 / k^{2}$ and $\epsilon$ is one of the numbers $1,2, \cdots, 1 / k$.

We proceed inductively. Suppose $\phi_{n_{1}}, \cdots, \phi_{n_{k-1}}$ have been chosen. Let $I_{\nu}=\left[-2^{v}, 2^{v}\right]$. For each $N<i$, and $\epsilon$ a member of $\{1,1 / 2, \cdots, 1 / k\}$ we consider the mapping $T_{N, \mathrm{e}}$ of $I_{1} \times I_{2} \times \cdots \times I_{i}$ into the metric space $\mathrm{Tl}$ of all measurable subsets of $[0,1]$ defined as follows:

$$
T_{N, \epsilon}\left(a_{1}, \cdots, a_{i}\right)=e_{i, N, \epsilon} .
$$

Clearly this mapping is continuous. Hence the image of $I_{1} \times \cdots \times I_{i}$ under $T_{N, \epsilon}$ is totally bounded.

For fixed $j, i<j \leqq k-1$ we consider

$$
f_{s, j}\left(a_{1}, \cdots, a_{i}\right)=g_{s, j}\left(e_{i, N, \epsilon}\right)=\int_{e_{i, N, \epsilon}} \phi_{n_{j}} \phi_{s} / f^{2}(x) d x
$$

and

$$
\tilde{f}_{s}\left(a_{1}, \cdots, a_{i}\right)=\tilde{g}_{8}\left(e_{i, N, \epsilon}\right)=\int_{e_{i, N, \epsilon}} \phi_{s}^{2} / f^{2}(x) d x .
$$

Clearly $g_{s, j}$ tends pointwise to zero and $\tilde{g}_{s}$ tends pointwise to $\left|e_{i, N, \epsilon}\right|$. It is easy to see that if $G, H$ are any elements of $\mathscr{T}$ and $\alpha$ has the meaning of Lemma 1,

$$
\begin{gathered}
\left|g_{8, j}(G)-g_{8, j}(H)\right| \leqq \alpha^{2}|G \Delta H|, \\
\left|\tilde{g}_{s}(G)-\tilde{g}_{8}(H)\right| \leqq \alpha^{2}|G \Delta H| .
\end{gathered}
$$

The above two inequalities imply that $\left\{g_{s, j}\right\}$ and $\left\{\tilde{g}_{s}\right\}$ are uniformly equi- 
continuous. But a pointwise convergent sequence of uniformly equicontinuous functions is uniformly convergent. Therefore for $s$ large enough,

$$
\left|g_{s, j}\left(e_{i, N, \epsilon}\right)\right|=\left|\int_{e_{i, N, \ell}} \phi_{n_{j}} \phi_{s} / f^{2}(x) d x\right| \leqq \frac{1}{4^{k}} \frac{1}{k^{2}} \leqq \frac{1}{4^{k}}\left|e_{i, N, \epsilon}\right|
$$

and

$$
\left|\tilde{g}_{8}\left(e_{i, N, \epsilon}\right)\right|=\left|\int_{e_{i, N, \epsilon}} \phi_{s}^{2} / f^{2}(x) d x\right| \leqq\left(1+\frac{1}{4}\left|e_{i, N, \epsilon}\right|\right) .
$$

Since for a fixed $k$ there are only a finite number of values of $i, N$, $\epsilon$, and $j$ under consideration, it follows that for $s$ larger than, say, $s_{0}$ inequalities (31) and (32) hold for all permissible $i, N, \epsilon$, and $j$. Hence we pick $\phi_{n_{k}}$ such that $n_{k}>s_{0}$.

We will now show that if $\left\{\phi_{n_{k}}\right\}$ is chosen to satisfy Lemmas 1, 2, and 3 it will satisfy (7), the inequality we are trying to prove. We have four lemmas.

Lemma 4. Let $\theta$ be a fixed number greater than 1 and let $N_{j}$ be a sequence of integers satisfying

$$
\theta^{j} \leqq B_{N_{j}}^{2}<\theta^{j+1}
$$

(for $j$ large enough, $N_{j}$ clearly exists). Let $\epsilon^{\prime}$ be any number greater than zero and let

$$
\Gamma_{j}=\left\{x \in F: S_{N_{j}}(x)>f(x)\left(1+\epsilon^{\prime}\right)\left(2 A_{N_{j}}^{2} \log \log A_{N_{j}}\right)^{1 / 2}\right\} .
$$

Then

$$
\sum_{j=1}^{\infty}\left|\Gamma_{j}\right|<\infty
$$

By Lemma 1,

$$
\int_{F} \exp \left(\frac{\lambda S_{N}(x)}{f(x)}\right) d x \leqq \frac{3}{2}|F| \exp \left(\frac{\lambda^{2} A_{N_{j}}^{2}}{2}\left(1+\epsilon^{\prime}\right)\right)
$$

provided $\lambda$ is small enough. If we let $\lambda=\left(2 A_{N j}^{-2} \log \log A_{N_{j}}\right)^{1 / 2}$, then for $j$ large enough the hypotheses of Lemma 1 are satisfied and (36) holds. We let $y=\left(1+\epsilon^{\prime}\right)\left(2 A_{N_{j}}^{2} \log \log A_{N_{j}}\right)^{1 / 2}$. Hence

$$
\left|\Gamma_{j}\right|=\left|\left\{x: S_{N_{j}}(x)>f(x) y, x \in F\right\}\right| \text {. }
$$

Clearly

$$
e^{\lambda y}\left|\Gamma_{j}\right| \leqq \int_{F} \exp \left(\frac{\lambda S_{N}}{f(x)}\right) d x \leqq \frac{3}{2}|F| \exp \left(\frac{\lambda^{2} A_{N_{i}}^{2}}{2}\left(1+\epsilon^{\prime}\right)\right) .
$$


Thus

$$
\begin{aligned}
\left|\Gamma_{j}\right| & \leqq \frac{3}{2}|F| \exp \left(\frac{\lambda A_{N_{j}}^{2}}{2}\left(1+\epsilon^{\prime}\right)-\lambda y\right) \\
& \leqq \frac{3}{2}|F| \exp \left(-\left(1+\epsilon^{\prime}\right) \log \log A_{N_{j}}\right) \\
& \leqq \frac{3}{2}|F|(\log \theta)^{-1-\epsilon^{\prime} j^{-1-\epsilon^{\prime}}}
\end{aligned}
$$

which implies

$$
\sum_{j=1}^{\infty}\left|\Gamma_{j}\right|<\infty
$$

Lemma 5. If $\epsilon, \theta$, and $N_{j}$ satisfy the conditions of Lemma 4 and

$$
\Gamma_{j}^{\prime}=\left\{x \in E: S_{N_{j}}(x)>\epsilon^{\prime}\left(2 A_{N_{j}}^{2} \log \log A_{N_{j}}\right)^{1 / 2}\right\}
$$

then

$$
\sum_{j=1}^{\infty}\left|\Gamma_{j}^{\prime}\right|<\infty .
$$

If $y=\epsilon^{\prime}\left(2 A_{N_{j}}^{2} \log \log A_{N_{j}}\right)^{1 / 2}$ then $\Gamma_{j}^{\prime}=\left\{x \in E: S_{N}(x)>\epsilon^{\prime} y\right\}$. We take $\lambda=\left(2 / \epsilon^{\prime}\right)\left(2 A_{N_{j}}^{-2} \log \log A_{N j}\right)^{1 / 2}, \eta=\epsilon^{2} / 4$. For $j$ large enough the conditions of Lemma 2 are satisfied and hence

$$
\int_{E} \exp \left(\lambda S_{N_{j}}\right) d x<2 \exp \left(\eta \lambda^{2} A_{N_{j}}^{2}\right)
$$

Clearly

$$
e^{\lambda y}\left|\Gamma_{j}^{\prime}\right| \leqq \int_{E} \exp \left(\lambda S_{N_{j}}\right) d x \leqq 2 \exp \left(\eta \lambda^{2} A_{N_{j}}^{2}\right)
$$

and hence

$$
\left|\Gamma_{j}^{\prime}\right| \leqq \exp \left(\eta \lambda^{2} j A_{N_{j}}^{2}-\lambda y\right) \leqq \exp \left(-2 \log \log A_{N_{j}}\right) \leqq(\log \theta)^{-2} j^{-2} .
$$

Thus

$$
\sum_{j=1}^{\infty}\left|\Gamma_{j}^{\prime}\right|<\infty .
$$

We now proceed to show that for the subsequence we have chosen that 


$$
\lim \sup \frac{S_{N}(x)}{\left(2 A_{N}^{2} \log \log A_{N}\right)^{1 / 2}}=f(x)
$$

almost everywhere in $F$. This is clearly implied by the following lemma.

Lемма 6. If $\epsilon$ is any number of the form $1 / n$ for $n$ a positive integer, $N_{j}$ $=N_{j}(\theta)$ a sequence of integers satisfying (33), and if

$$
\begin{aligned}
& \tilde{S}_{N_{j}}^{*}=\sup _{N_{j-1}<i \leqq N_{j}} S_{i}(x), \\
& \tilde{\Gamma}_{j}=\left\{x: \tilde{S}_{N_{j}}^{*}(x)>f(x)(1+\epsilon)\left(2 A_{N_{j}}^{2} \log \log A_{N_{j}}\right)^{1 / 2}\right\}
\end{aligned}
$$

then there exist a $\theta$ for which

Clearly

$$
\sum_{j=1}^{\infty}\left|\tilde{\Gamma}_{j}\right|<\infty
$$

$$
\begin{array}{r}
\tilde{\Gamma}_{j} \subset\left\{x: \tilde{S}_{N_{j}}^{*}(x)>f(x)(1+\epsilon)\left(2 A_{N_{j-1}}^{2} \log \log A_{N_{j-1}}\right)^{1 / 2}\right\} \\
=\bigcup_{p=N_{j-1}+1}^{N_{j}} e_{N_{j-1}, p, \epsilon}=\bigcup_{p=N_{j-1}+1}^{N_{j}} e_{p}
\end{array}
$$

where $e_{N_{j-1}, p, \epsilon}$ has the same meaning as in Lemma 3 . Hence

$$
\left|\tilde{\Gamma}_{j}\right| \leqq \sum_{p=N_{j-1}+1}^{N_{j}}\left|e_{p}\right|
$$

Now we consider those $p$ for which $\left|e_{p}\right|>1 / p^{2}$. If $\sigma_{p}(x)=S_{N_{j}}(x)-S_{p}(x)$ then $S_{N_{j}}(x) \geqq S_{p}(x)-\left|\sigma_{p}(x)\right|$ and thus

$$
\begin{aligned}
\mid\{x: x & \left.\in e_{p}, S_{N_{j}}(x) / f(x)>(1+\epsilon)\left(2 A_{N_{j-1}}^{2} \log \log A_{N_{j-1}}\right)^{1 / 2}-(2)^{1 / 2} A_{N_{j-1}}\right\} \mid \\
& \geqq\left|e_{p}\right|-\left|\left\{x: x \in e_{p}, \sigma_{p}(x) / f(x)>(2)^{1 / 2} A_{N_{j-1}}\right\}\right|=\left|e_{p}\right|-\left|d_{p}\right| .
\end{aligned}
$$

To estimate $\left|d_{p}\right|$ we write

$$
\int_{e_{p}}\left(\sigma_{p}(x) / f(x)\right)^{2} d x=\int_{e_{p}}\left(\left(\sum_{p+1}^{N_{j}} a_{k} \phi_{k}\right) / f(x)\right)^{2} d x .
$$

Applying Lemma 3 we see that if $N_{j}$ is large enough then

$$
\begin{aligned}
\int_{e_{p}} & \left(\left(\sum_{p+1}^{N_{j}} a_{k} \phi_{k}\right) / f(x)\right)^{2} d x \\
= & \sum_{p+1}^{N_{j+1}} a_{k}^{2} \int_{e_{p}}\left(\phi_{k} / f(x)\right)^{2} d x+\sum_{i \neq k} a_{i} a_{k} \int_{e_{p}}\left(\phi_{i} \phi_{k} / f^{2}(x)\right) d x \\
& \leqq \sum_{p+1}^{N_{j}} a_{k}^{2}\left|e_{p}\right|(1+1 / 4)+2 \sum_{k=p+1}^{N_{j}} \frac{a_{k}}{2^{k}} \sum_{i=p+1}^{k-1} \frac{a_{i}}{2^{k}}\left|e_{p}\right| \leqq \sum_{p+1}^{N_{j}} a_{k}^{2}\left|e_{p}\right|(1+1 / 2) .
\end{aligned}
$$


Clearly,

$$
\sum_{p+1}^{N_{j}} a_{k}^{2} \leqq A_{N_{j}}^{2}-A_{N_{j-1}}^{2} \leqq \theta^{j+1}-\theta^{j-1}=\theta^{j-1}\left(\theta^{2}-1\right) .
$$

Now if $\theta$ is close enough to $1, A_{N j-1}^{2} \geqq \theta^{j-1} \geqq \theta^{j-1}\left(\theta^{2}-1\right)$ and hence

$$
\int_{e_{p}}\left(\sigma_{p}(x) / f(x)\right)^{2} d x \leqq \sum_{p+1}^{N i} a_{k}^{2}\left|e_{p}\right|\left(1+\frac{1}{2}\right) \leqq \frac{3}{2}\left|e_{p}\right| A_{N_{j-1}} .
$$

Thus clearly

$$
2\left|d_{p}\right| A_{N_{j-1}}^{2} \leqq \int_{e_{p}}\left(\sigma_{p}(x) / f(x)\right)^{2} d x \leqq \frac{3}{2}\left|e_{p}\right| A_{N_{j-1}}^{2},
$$

which implies

$$
\left|d_{p}\right| \leqq \frac{3}{4}\left|e_{p}\right|
$$

Collecting the last few inequalities we see that

$$
\begin{aligned}
\left|\Delta_{j}\right| & =\bigcup_{p=N_{j-1}+1}^{N_{j}}\left\{x: x \in e_{p}, S_{N_{j}}(x) / f(x)\right. \\
& \left.>(1+\epsilon)\left(2 A_{N_{j-1}}^{2} \log \log A_{N_{j-1}}\right)^{1 / 2}-(2)^{1 / 2} A_{N_{j-1}}\right\} \\
& \geqq \frac{1}{4} \sum\left|e_{p}\right|
\end{aligned}
$$

where the sum is taken over all $p$ for which $\left|e_{p}\right|>1 / p^{2}, N_{j-1}+1 \leqq p \leqq N_{j}$. Since

$$
\sum_{j>j_{0}}\left|\tilde{\Gamma}_{j}\right| \leqq \sum_{p=1}^{\infty} p^{-2}+4 \sum_{j>j_{0}}\left|\Delta_{j}\right|
$$

we now must estimate $\left|\Delta_{j}\right|$.

If $j$ is large enough then $\left(\log \log A_{N_{j-1}}\right)^{1 / 2}>\epsilon / 2$ which gives

$$
\begin{aligned}
(1+\epsilon)\left(2 A_{N_{j-1}}^{2} \log \log A_{N_{j-1}}\right)^{1 / 2}- & (2)^{1 / 2} A_{N_{j-1}} \\
& \geqq(1+\epsilon / 2)\left(2 A_{N_{j-1}}^{2} \log \log A_{N_{j-1}}\right)^{1 / 2} .
\end{aligned}
$$

Clearly

$$
\frac{\log \log A_{N_{j-1}}}{\log \log A_{N_{j}}} \rightarrow 1
$$

and

$$
\frac{A_{N_{j-1}}}{A_{N_{j}}} \geqq\left(\frac{\theta^{j-1}}{\theta^{j+1}}\right)^{1 / 2}=\frac{1}{\theta}
$$


Hence if $j$ is large enough and $\theta$ is so close to 1 that $(1+\epsilon / 2) \theta^{-2}>(1+\epsilon / 4)$ we have

$(1+\epsilon / 2)\left(2 A_{N_{j-1}}^{2} \log \log A_{N_{j-1}}\right)^{1 / 2}$

$=(1+\epsilon / 2)\left(2 A_{N_{j}}^{2} \log \log A_{N_{j}}\right)^{1 / 2}\left(\frac{2 A_{N_{j-1}}^{2} \log \log A_{N_{j-1}}}{2 A_{N_{j}}^{2} \log \log A_{N_{j}}}\right)^{1 / 2}$

$\geqq(1+\epsilon / 2) \theta^{-2}\left(2 A_{N_{j}}^{2} \log \log A_{N_{j}}\right)^{1 / 2} \geqq(1+\epsilon / 4)\left(2 A_{N_{j}}^{2} \log \log A_{N_{j}}\right)^{1 / 2}$.

The above inequality and inequality (40) imply

$$
\left|\Delta_{j}\right| \leqq\left|\left\{x: S_{N_{i}}(x) \geqq(1+\epsilon / 4)\left(2 A_{N}^{2}, \log \log A_{N_{j}}\right)^{1 / 2}\right\}\right| .
$$

Taking $\epsilon^{\prime}=\epsilon / 4$ and applying Lemma 4 we see that

$$
\sum_{j=1}^{\infty}\left|\Delta_{j}\right|<\infty,
$$

which together with (39) gives us the conclusion of the lemma.

The following lemma easily implies that

$$
\lim \frac{S_{N}(x)}{\left(2 A_{N}^{2} \log \log A_{N}\right)^{1 / 2}}=0
$$

almost everywhere in $E$ and hence completes Part I of this paper.

LEMMA 7. If $\epsilon$ is any number of the form $1 / n$ for $n$ a positive integer, $N_{j}$ $=N_{j}(\theta)$ a sequence of integers satisfying (33), and if

$$
\begin{aligned}
\tilde{S}_{N_{j}}^{*}(x) & =\sup _{N_{j-1} \geqq_{i} \leqq N_{i}} S_{i}(x), \\
\tilde{\Gamma}_{j}^{\prime} & =\left\{x: \widetilde{S}_{N_{j}}^{*}(x)>\epsilon\left(2 A_{N_{j}}^{2} \log \log A_{N_{j}}\right)^{1 / 2}, x \in E\right\}
\end{aligned}
$$

then

$$
\sum_{1}^{\infty}\left|\tilde{\Gamma}_{j}^{\prime}\right|<\infty .
$$

Arguing just as in Lemma 6 we obtain

$$
\begin{aligned}
\tilde{\Gamma}_{j}^{\prime} & \subset\left\{x: x \in E, \tilde{S}_{N_{j}}^{*}(x)>\epsilon\left(2 A_{N_{j-1}}^{2} \log \log A_{N_{j-1}}\right)^{1 / 2}\right\} \\
& =\bigcup_{p=N_{j-1}+1}^{N_{j}} e_{N_{j-1}, p, \epsilon}^{\prime}=U e_{p}^{\prime},
\end{aligned}
$$

and hence for $j$ sufficiently large and $\left|e_{p}^{\prime}\right|>p^{-2}$,

$$
\left|\left\{x: x \in e_{p}^{\prime}, S_{N_{j}}(x)>\epsilon\left(2 A_{N_{j-1}}^{2} \log \log A_{N_{j-1}}\right)^{1 / 2}-2^{1 / 2} A_{N_{j-1}}\right\}\right| \geqq \frac{1}{2}\left|e_{p}^{\prime}\right| .
$$


Thus if

$$
\Delta_{j}^{\prime}=\left\{x: S_{N_{j}}(x)>\epsilon\left(2 A_{N_{j-1}}^{2} \log \log A_{N_{j-1}}\right)^{1 / 2}-2^{1 / 2} A_{N_{j-1}} ; x \in E\right\},
$$

we have

$$
\sum_{j \geq j_{0}}\left|\tilde{\Gamma}_{j}^{\prime}\right| \leqq 2 \sum_{j \geq j_{0}}\left|\Delta_{j}^{\prime}\right|+\sum_{1}^{\infty} p^{-2}
$$

Hence we need only show that

$$
\sum_{j=j_{0}}\left|\Delta_{j}^{\prime}\right|<\infty .
$$

If $j$ is so large that $1<(\epsilon / 2)\left(\log \log A_{N_{j-1}}\right)^{1 / 2}$ then

$$
\epsilon\left(2 A_{N_{j-1}}^{2} \log \log A_{N_{j-1}}\right)^{1 / 2}-2^{1 / 2} A_{N_{j-1}} \geqq(\epsilon / 2)\left(2 A_{N_{j-1}}^{2} \log \log A_{N_{j-1}}\right)^{1 / 2}
$$

Arguing as in Lemma 6 we see that if $\theta$ is close enough to 1 , then

$$
(\epsilon / 2)\left(2 A_{N_{j-1}}^{2} \log \log A_{N_{j-1}}\right)^{1 / 2} \geqq(\epsilon / 4)\left(2 A_{N_{j}}^{2} \log \log A_{N_{j}}\right)^{1 / 2},
$$

and hence

$$
\left|\Delta_{j}^{\prime}\right| \leqq\left|\left\{x: S_{N_{j}}(x) \geqq(\epsilon / 4)\left(2 A_{N_{j}}^{2} \log \log A_{N_{j}}\right)^{1 / 2}\right\}\right| .
$$

Applying Lemma 5 with $\epsilon / 4=\epsilon^{\prime}$ we obtain

$$
\sum_{j \geq j_{0}}\left|\Delta_{j}^{\prime}\right|<\infty .
$$

This completes the proof of Lemma 7.

As was remarked above, Lemmas 6 and 7 imply

$$
\lim \sup \frac{S_{N}(x)}{\left(2 A_{N}^{2} \log \log A_{N}\right)^{1 / 2}} \leqq f(x)
$$

a.e. in $E \cup F$, which is what we wished to prove. However we should note that we have proved (41) only for sequences $\left\{a_{k}\right\}$ satisfying $-2^{k} \leqq a_{k} \leqq 2^{k}$. This is not really a restriction, however since it is clear that for any $\left\{a_{k}\right\}$ satisfying conditions (3) of the theorem, there exists a constant $c$ for which $-2^{k} \leqq c a_{k} \leqq 2^{k}$ for all $j$.

Part II. This part of the paper will be devoted to showing that we can choose $\left\{\phi_{n_{k}}\right\}$ for which

$$
\lim \sup \frac{S_{N}(x)}{\left(2 A_{N}^{2} \log \log A_{N}\right)^{1 / 2}} \geqq f(x) .
$$

It should be noted that in Part I, for the set $E$ on which $f(x)=0$, we demonstrated (41) with lim in place of lim sup. Therefore it suffices to show that 
(42) holds on every set $F$ on which $f(x)$ is bounded away from zero.

We first choose $\left\{\phi_{n_{k}}\right\}$ by induction and then show that it satisfies (42). We suppose that $\phi_{n_{1}}, \cdots, \phi_{n_{k-1}}$ have been chosen.

We begin by defining a mapping $T_{e, P}$ of $I_{1} \times I_{2} \times \cdots \times I_{j}$ into the metric space $T$ of all measurable subsets of $[0,1]$ where $\epsilon$ is any number greater than zero, $P$ is any partition of $(1,2, \cdots, j)$, and $I_{i}=\left[-2^{i}, 2^{i}\right]$. Let $1<i_{1}$ $<i_{2}<\cdots<i_{s+1}=j$ be the sequence of integers defining $P$. For each point $\left(a_{1}, \cdots, a_{j}\right)$ of $I_{1} \times I_{2} \times \cdots \times I_{j}$ we consider the $s+1$ tuple of functions

$$
\left(\frac{S_{i_{1}}(x)}{f(x)}, \frac{S_{i_{2}}(x)-S_{i_{1}}(x)}{f(x)}, \ldots, \frac{S_{i_{+1}}(x)-S_{i_{0}}(x)}{f(x)}\right)
$$

where $S_{i_{p}}(x)$ is of course $\sum_{i=1}^{p} a_{l} \phi_{n_{l}}(x)$. Consider the following sets

$$
\begin{aligned}
& E_{1}=\left\{x: x \in F ; S_{i_{1}}(x)>f(x)(1-\epsilon)\left(2 A_{i_{1}}^{2} \log \log A_{i_{1}}\right)^{1 / 2}\right\}, \\
& E_{2}=\left\{x: x \in F-E_{1} ; S_{i_{2}}(x)-S_{i_{1}}(x)\right. \\
& \left.>f(x)(1-\epsilon)\left(2\left(A_{i_{2}}^{2}-A_{i_{1}}^{2}\right) \log \log \left(A_{i_{2}}^{2}-A_{i_{1}}^{2}\right)\right)^{1 / 2}\right\}, \\
& E_{s+1}=\left\{x: x \in F-\left(E_{1}+\cdots+E_{s}\right) ; S_{j}(x)-S_{i_{s}}(x)\right. \\
& \left.>f(x)(1-\epsilon)\left(2\left(A_{j}^{2}-A_{i_{s}}^{2}\right) \log \log \left(A_{j}^{2}-A_{i s}^{2}\right)\right)^{1 / 2}\right\} .
\end{aligned}
$$

We then define

$$
T_{\epsilon, P}\left(a_{1}, \cdots, a_{j}\right)=F_{\left(a_{1}, \cdots, a_{j}\right)}^{\epsilon, P}=F-\left(E_{1}+\cdots+E_{s+1}\right) .
$$

We have the following lemma. (As in Part I, we write $\psi_{n}=\phi_{n} / f(x)$.)

Lemma 8. Let $\epsilon, P, j$, and $k$, where $j<k$, be fixed. Then the functions

$$
f_{N}\left(a_{1}, \cdots a_{j}\right)=g_{N}\left(F_{a_{1}}, \cdots, a_{j}\right)=\sum_{\left(\beta_{1}, \cdots, \beta_{k-j-1}\right)}\left|\int \psi_{n_{j+1}}^{\beta_{1}} \cdots \psi_{n_{k-1}}^{\beta_{k-j-1}} \psi_{N} d x\right|,
$$

and

$$
\begin{aligned}
\tilde{f}_{N}\left(a_{1}, \cdots, a_{j}\right) & =\tilde{g}_{N}\left(F_{a_{1}}, \ldots, a_{j}\right) \\
& =\sum_{\left(\beta_{1}, \cdots, \beta_{k-j-1}\right)}\left|\int \psi_{n_{j+1}}^{\beta_{1}} \cdots \psi_{n_{k-1}}^{\beta_{k-j-1}} \psi_{N}^{2}-\psi_{n_{j+1}}^{\beta_{1}} \cdots \psi_{n_{k-1}}^{\beta_{k-j-1}} d x\right|
\end{aligned}
$$

where the sums are taken over all $k-j-1$ tuples with elements from $\{0,1,2\}$, and the integrals are taken over the set $F_{a_{1}, \cdots, a_{i}}^{e, P}$ converge uniformly to zero on $I_{1} \times I_{2} \times \cdots \times I_{j}$ as $N \rightarrow \infty$.

Clearly $T_{\epsilon, P}$ is continuous. Since $I_{1} \times \cdots \times I_{j}$ is compact, its image in $\mathfrak{T}$ under $T_{\epsilon, P}$ is totally bounded. Now $g_{N}$ and $\tilde{g}_{N}$ are uniformly equicontinuous sequences of functions. For 


$$
\begin{aligned}
& \left|g_{N}(G)-g_{N}(H)\right| \leqq|G \Delta H| \alpha^{2(k-j)} 3^{k-j}, \\
& \left|\tilde{g}_{N}(G)-\tilde{g}_{N}(H)\right| \leqq|G \Delta H|(2 \alpha)^{2(k-j)} 3^{k-j}
\end{aligned}
$$

where $\alpha$ has the meaning of Lemma 1. Clearly $g_{N}$ and $\tilde{g}_{N}$ tend pointwise to zero. But it is easy to see that if a uniformly equicontinuous sequence of functions on a totally bounded metric space tends pointwise to zero it tends to zero uniformly.

We now choose $\phi_{n_{k}}$. We are going to choose it in such a way that we will be able to use Lemma 1 of Part I for certain sets $G$ and certain blocks of terms of our series. We assume now that $\phi_{n_{j}}$ has been chosen for all $j<k$. For each $j<k$ we consider all possible partitions of $(1,2, \cdots, j)$ and the following values of $\epsilon: \epsilon=1,1 / 2, \cdots, 1 / k$. For each such $\epsilon$ and each partition we apply the preceding lemma and find an $N_{0}(P, \epsilon)$ so large that if $N>N_{0}(P, \epsilon)$ then

$$
\begin{aligned}
& f_{N}^{P, \epsilon}\left(a_{1}, \cdots, a_{j}\right)<\frac{1}{k} \epsilon_{k}^{\prime}, \\
& \tilde{f}_{N}^{P, \epsilon}\left(a_{1}, \cdots, a_{j}\right)<\frac{1}{k} \epsilon_{k}
\end{aligned}
$$

where $\left\{\epsilon_{k}\right\}$ and $\left\{\epsilon_{k}^{\prime}\right\}$ are defined in the proof of Lemma 1. Since for fixed $k$ there are only a finite number of partitions and a finite number of values of $\epsilon$ allowed, we can pick $N_{0}$ so large that for $N>N_{0}$ (43) and (44) hold for all these values of $P$ and $\epsilon$. We then pick $n_{k}$ to be any integer greater than $N$.

From now on we denote our fixed subsequence $\left\{\phi_{n_{k}}\right\}$ by $\left\{\phi_{n}\right\}$.

The following lemma contains the major part of the proof of Part II.

LEMMA 9. Let

$$
S(x)=\sum_{N_{1}}^{N_{2}} a_{n} \phi_{n}(x), \quad A^{2}=\sum_{N_{1}}^{N_{2}} a_{n}^{2}, \quad \lambda=20\left(2 A^{-2} \log \log A\right)^{1 / 2} .
$$

Let $\epsilon$ be one of the numbers $1,1 / 2, \cdots, 1 / k$, and $F$ be $F_{a_{1}, \cdots, a_{N_{1}}}^{e, P}$ for some partition $P$. Then there exists an $\eta$ depending only on $\epsilon$ such that if the hypotheses (9) of Lemma 1 are satisfied and if $A$ is larger than some $A_{0}$ depending only on $\epsilon$ and if furthermore

$$
|F| \geqq 1 / N_{1}
$$

then

$$
W\left((1-\epsilon)\left(2 A^{2} \log \log A\right)^{1 / 2}\right) \geqq|F|(\log A)^{-1},
$$

where

$$
W(y)=|\{x: x \in F, S(x) / f(x)>y\}| .
$$

Inequalities (43) and (44) together with $|F| \geqq 1 / N$, imply 


$$
f_{n}\left(a_{1}, \cdots, a_{N_{1}-1}\right) \leqq \epsilon_{n}^{\prime}|F|,
$$

and

$$
\tilde{f}_{n}\left(a_{1}, \cdots, a_{N_{1}-1}\right) \leqq \epsilon_{n}|F| .
$$

We note that the above two inequalities are identical with (14) and (15) of Lemma 1, which were the only conditions needed on the subsequence for the lemma to hold. Also the hypotheses of our lemma imply the hypotheses of Lemma 1. Therefore

$$
\begin{aligned}
\frac{1}{2}|F| \exp \left(\frac{\lambda^{2} A^{2}}{2}(1-\eta)\right) & \leqq \int_{F} \exp \left(\lambda \frac{S(x)}{f(x)}\right) d x \\
& \leqq \frac{3}{2}|F| \exp \left(\frac{\lambda^{2} A^{2}}{2}(1+\eta)\right) .
\end{aligned}
$$

For the remainder of this lemma we will denote $S(x) / f(x)$ by $S(x)$.

We take the following values of $\eta, \delta, \epsilon^{\prime}$, and $a$ :

$$
\begin{aligned}
\left(1-\epsilon^{\prime}\right)^{2}(1+4 \delta) & =1, \quad\left(1-\epsilon^{\prime}\right)(1-\delta)=1-\epsilon, \quad \eta=\delta^{2} / 8, \\
a & =\left(1-\epsilon^{\prime}\right)\left(2 A^{-2} \log \log A\right)^{1 / 2} .
\end{aligned}
$$

We consider

$$
\begin{aligned}
\int_{F} e^{a \hat{S}(x)} d x & =-\int_{-\infty}^{\infty} e^{a y} d W(y)=a \int_{-\infty}^{\infty} e^{a y} W(y) d y \\
& =a\left(\int_{-\infty}^{0}+\int_{0}^{a A^{2}(1-\delta)}+\int_{a A^{2}(1-\delta)}^{a A^{2}(1+\delta)}+\int_{a A^{2}(1+\delta)}^{8 a A^{2}} \int_{8 a A^{2}}^{\infty}\right) \\
& =a\left(J_{1}+J_{2}+J_{3}+J_{4}+J_{5}\right) .
\end{aligned}
$$

First we will show that

$$
a\left(J_{1}+J_{2}+J_{4}+J_{5}\right)<(5 / 6) \int_{F^{\prime}} e^{a \hat{s}} d x,
$$

and hence

$$
a J_{3}>(1 / 6) \int_{F} e^{a \hat{s}} d x .
$$

This latter inequality implies, by Lemma 1 ,

$$
a J_{3}>(1 / 12)|F| \exp \left(\frac{a^{2} A^{2}}{2}(1-\eta)\right) .
$$

It is this inequality which we will then use to obtain the estimate for $W\left((1-\epsilon)\left(2 A^{2} \log \log A\right)^{1 / 2}\right)$. 
Estimation of a $J_{1}$ : Clearly,

$$
a J_{1}=a \int_{-\infty}^{0} e^{a y w}(y) d y \leqq a \int_{-\infty} e^{a y}|F| d y \leqq|F| .
$$

Estimation of $a J_{5}:$ We let $\lambda=8 a$ and apply Lemma 1 . Hence

$$
W(y) e^{\lambda y} \leqq \int e^{\lambda \hat{S}} d x \leqq \frac{3}{2}|F| \exp \left(\frac{\lambda^{2} A^{2}}{2}(1+\eta)\right)
$$

which implies $W(y) \leqq(3 / 2)|F| \exp \left((1+\eta) 32 a^{2} A^{2}-8 a y\right)$. If $y \geqq 8 a A^{2}$ then

$$
(1+\eta) 32 a^{2} A^{2}-8 a y \leqq 4(1+\eta) a y-8 a y \leqq-2 a y,
$$

and hence $W(y) \leqq(3 / 2)|F| e^{-2 a y}$. This implies

$$
a J_{5} \leqq \frac{3}{2}|F| a \int_{8 a A^{2}}^{\infty} e^{-a y} d y \leqq \frac{3}{2}|F| .
$$

Estimation of $a J_{2}$ and $a J_{4}$ : Let $\lambda=y A^{-2}$. Since in $J_{2}$ and $J_{4}, y \leqq 8 a A^{2}$ $\leqq 8\left(2 A^{2} \log \log A\right)^{1 / 2}$, we have $\lambda \leqq 8\left(2 A^{-2} \log \log A\right)^{1 / 2}$ which allows us to apply Lemma 1 . Thus

$$
\begin{aligned}
W(y) & \leqq \frac{3}{2}|F| \exp \left(\frac{\lambda^{2} A^{2}}{2}(1+\eta)-\lambda y\right) \leqq \frac{3}{2}|F| \exp \left(-(1-\eta) y^{2} A^{-2} / 2\right) \\
& \leqq \frac{3}{2}|F| \exp \left(-\left(1-\delta^{2} / 8\right) y^{2} B^{-2} / 2\right),
\end{aligned}
$$

and hence

$$
a\left(J_{2}+J_{4}\right) \leqq a\left(\int_{0}^{a A^{2}(1-\delta)}+\int_{a A^{2}(1+\delta)}^{8 a A^{2}} \frac{3}{2}|F| \exp \left(a y-\left(1-\delta^{2} / 8\right) y^{2} A^{-2} / 2\right) d y\right) \mathbf{a}
$$

The maximum of the function $a y-\left(1-\delta^{2} / 8\right) y A^{-2} / 2$ is located at the point $y=a A^{2}\left(1-\delta^{2} / 8\right)^{-1}$. Hence in the interval $\left(a A^{2}(1+\delta), 8 a A^{2}\right)$, $a y$ $-\left(1-\delta^{2} / 8\right) y^{2} A^{-2} / 2$ is majorized by its value at $y=a A^{2}(1+\delta)$ which is

$$
a^{2} A^{2}(1+\delta)-\left(1-\delta^{2} / 8\right)(1+\delta)^{2} a^{2} A^{2} / 2 \leqq\left(1-\delta^{2} / 2\right) a^{2} A^{2} / 2 .
$$

Similarly, in the interval $\left(0, a A^{2}(1-\delta)\right)$ we have $a y-\left(1-\delta^{2} / 8\right) y^{2} A^{-2} / 2$ $\leqq\left(1-\delta^{2} / 2\right) a^{2} A^{2} / 2$. Hence

$$
a\left(J_{2}+J_{4}\right) \leqq 12|F| a^{2} A^{2} \exp \left(\left(1-\delta^{2} / 2\right) a^{2} A^{2} / 2\right) .
$$

If $A$ is so large that $12 a^{2} A^{2} \leqq(3 / 16) e^{a^{2} A^{22} / 8}$, the above equation and Lemma 1 give us

$$
a\left(J_{2}+J_{4}\right) \leqq \frac{3}{16}|F| \exp \left(\left(1-\delta^{2} / 4\right) a^{2} A^{2} / 2\right) \leqq \frac{1}{2} \int_{F} e^{a \hat{s}} d x .
$$


If $A$ is so large that $15|F| \leqq|F| \exp \left((1-\eta) a^{2} A^{2} / 2\right)$, then equations (48) and (49) together with Lemma 1 give us

$$
a\left(J_{1}+J_{6}\right) \leqq \frac{5}{2}|F| \leqq \frac{1}{6}|F| \exp \left((1-\eta) a^{2} A^{2} / 2\right) \leqq \frac{1}{3} \int_{F} e^{a \hat{s}} d x
$$

Equations (51) and (52) imply

$$
a J_{3}>\frac{1}{6} \int_{F} e^{a \hat{s}} d x>\frac{1}{12}|F| \exp \left((1-\delta) a^{2} A^{2} / 2\right) .
$$

We now investigate $a J_{3}$ :

$$
a J_{3}=a \int_{a A^{2}(1-\delta)}^{a A^{2}(1+\delta)} e^{a v \varpi}(y) d y \leqq 2 a^{2} A^{2} \exp \left(a^{2} A^{2}(1+\delta)\right) w\left(a A^{2}(1-\delta)\right) .
$$

Hence from (53) it follows that

$$
\frac{1}{12}|F| \exp \left((1-\delta) a^{2} A^{2} / 2\right) \leqq 2 a^{2} A^{2} \exp \left(a^{2} A^{2}(1+\delta)\right) w\left(a A^{2}(1-\delta)\right) .
$$

This implies

$$
\rightsquigarrow\left(a A^{2}(1-\delta)\right) \geqq|F|\left(24 a^{2} A^{2}\right)^{-1} \exp \left(-(1+3 \delta) a^{2} A^{2} / 2\right) .
$$

If $A$ is so large that $\log 24 a^{2} A^{2}<a^{2} A^{2} \delta / 2$, then

$$
\begin{aligned}
W\left(a A^{2}(1-\delta)\right) & >|F| \exp \left(-(1+4 \delta) a^{2} A^{2} / 2\right) \\
& \geqq|F| \exp \left(-\left(1-\epsilon^{\prime}\right)^{2}(1+4 \delta) \log \log A\right) \\
& =|F|(\log A)^{-1} .
\end{aligned}
$$

Since $a A^{2}(1-\delta)=\left(1-\epsilon^{\prime}\right)(1-\delta)\left(2 A^{2} \log \log A\right)^{1 / 2}=(1-\epsilon)\left(2 A^{2} \log \log A\right)^{1 / 2}$, equation (54) implies the conclusion of the lemma.

We will prove the remaining part of the theorem in the following modified form.

Lemma 10. If $\epsilon>0, \delta>0$, are given (small) quantities, and $N_{0}$ is a given integer then there exists a finite sequence of integers $N_{0}<N_{1}<\cdots<N_{k}$ such that

$$
\max _{1 \leqq j \leqq k} \frac{S_{N_{j}}(x)}{\left(2 A_{N_{j}}^{2} \log \log A_{N_{j}}\right)^{1 / 2}} \geqq(1-\epsilon) f(x)
$$

for $x$ in a set of measure greater than $|\bar{F}|-\delta$ where $\bar{F}$ is the set on which $f(x)>0$.

The general idea of the proof of the above lemma is as follows.

We fix $N_{0}, \epsilon, \delta$ and begin by cutting up our series $\sum_{1}^{\infty} a_{\nu} \phi_{\nu}(x)$ into successive disjoint blocks of terms in a manner which we will now explain. Let 


$$
\tilde{A}_{N_{i}}=\left(\sum a_{v}\right)^{1 / 2}
$$

where the sum is taken over all the coefficients in the $j$ th block. Let $C$ be a large number to be chosen later. We define the first block of terms to be the first terms of the series taken until

$$
\tilde{A}_{N_{1}} \geqq C \text {. }
$$

We note that

$$
1 \leqq \tilde{A}_{N_{1}}^{2} / C^{2} \leqq \tilde{A}_{N_{1}-1}^{2} / C^{2}+a_{N_{1}}^{2} / C^{2} \leqq 1+\underset{a_{N_{1}}^{2} / C^{2}}{2}
$$

and since $a_{k} / A_{k} \rightarrow 0, a_{N_{1}}^{2} / C^{2}$ is very small if $C$ is large. Thus

$$
1 \leqq \tilde{A}_{N_{1}}^{2} / C^{2} \leqq 1+\gamma
$$

where $\gamma$ can be taken as small as we please if only $C$ is large enough.

The $j$ th block of terms starts where the $j-1$ st leaves off and is taken until

$$
1 \leqq \tilde{A}_{N_{j}} / C^{j} \text {. }
$$

Thus

$$
1 \leqq \tilde{A}_{N_{j}}^{2} / C^{2} \leqq 1+\gamma
$$

The $j$ th block of terms, and hence the number $N_{j}$, is thus defined inductively. We denote the sum of the $j$ th block by $F_{j}(x)$.

Clearly if $C$ is very large then in some sense the $j$ th block of terms is the major portion of the partial sum $S_{N_{j}}(x)$, and we would expect it to determine the behavior of $S_{N_{j}}(x)$.

To prove the lemma it is sufficient to show that for $C$ large enough the following three points hold:

There exists a $k$ for which

$$
\max _{1 \leqq j \leqq k} \frac{F_{j}(x)}{\left(2 \tilde{A}_{N_{j}}^{2} \log \log \tilde{A}_{N_{j}}\right)^{1 / 2}} \geqq f(x)(1-\epsilon / 4)
$$

for all $x$ in a set of measure greater than $|\bar{F}|-\delta / 2$,

$$
\frac{S_{N_{j-1}}(x)}{\left(2 A_{N_{j}}^{2} \log \log A_{N_{j}}\right)^{1 / 2}} \leqq \frac{\epsilon}{2} f(x)
$$

for all $j \leqq k$, and all $x$ in a set of measure greater than $|\bar{F}|-\delta / 2$,

$$
\left(\frac{2 \tilde{A}_{N_{j}}^{2} \log \log \tilde{A}_{N_{j}}}{2 A_{N_{j}}^{2} \log \log A_{N_{j}}}\right)^{1 / 2} \geqq 1-\frac{\epsilon}{4} .
$$

Since 


$$
\begin{aligned}
& \frac{S_{N_{j}}(x)}{\left(2 A_{N_{j}}^{2} \log \log A_{N_{j}}\right)^{1 / 2}} \\
& =\frac{F_{j}(x)}{\left(2 \widetilde{A}_{N_{j}}^{2} \log \log \tilde{A}_{N_{j}}\right)^{1 / 2}} \frac{\left(2 \tilde{A}_{N_{j}}^{2} \log \log \tilde{A}_{N_{j}}\right)^{1 / 2}}{\left(2 A_{N_{j}}^{2} \log \log A_{N_{j}}\right)^{1 / 2}}-\frac{S_{N_{j-1}}(x)}{\left(2 A_{N_{j}}^{2} \log \log A_{N_{j}}\right)^{1 / 2}},
\end{aligned}
$$

points (1), (2) and (3) imply

$$
\max _{1 \leqq j \geqq_{k}} \frac{S_{N_{j}}(x)}{\left(2 A_{N^{j}}^{2} \log \log A_{N_{j}}\right)^{1 / 2}} \geqq\left(1-\frac{\epsilon}{4}\right)\left(1-\frac{\epsilon}{4}\right) f(x)-\frac{\epsilon}{2} f(x) \geqq(1-\epsilon) f(x)
$$

is a set of measure greater than $|\bar{F}|-\delta$.

It is easy to see that (3) holds provided $C$ is large enough. We take up point (1). Let $\bar{F}_{0}$ be a set such that $f(x)$ is bounded from zero on $\bar{F}_{0}$ and $\left|\bar{F}_{0}\right|>|\bar{F}|-\delta / 4$. Let $p$ be the least integer for which $1 / p \leqq \epsilon / 4$. Let

$$
\begin{aligned}
& E_{1}=\left\{x: x \in \bar{F}_{0}, F_{1}(x)>f(x)(1-1 / p)\left(2 \tilde{A}_{N_{1}}^{2} \log \log \tilde{A}_{N_{1}}\right)^{1 / 2}\right\}, \\
& \dot{E}_{j}=\left\{x: x \in \bar{F}_{0}-\left(E_{1}+\cdots+E_{j-1}\right), F_{j}(x)\right. \\
& \left.\quad>f(x)(1-1 / p)\left(2 \tilde{A}_{N_{j}}^{2} \log \log \tilde{A}_{N_{j}}\right)^{1 / 2}\right\} .
\end{aligned}
$$

We wish to show that if $k$ is large enough then

$$
\left|\bar{F}_{0}-\left(E_{1}+\cdots+E_{k}\right)\right| \leqq \delta / 4 .
$$

This clearly will imply point (3). If

$$
\frac{1}{N_{1}} \leqq \frac{1}{p}
$$

and

$$
\left|\bar{F}_{0}-\left(E_{1}+\cdots+E_{j-1}\right)\right|>\frac{1}{N_{1}},
$$

and if furthermore $C$ is large enough, then the hypotheses of Lemma 9 are satisfied for

$$
\epsilon=\frac{1}{p}, \quad S(x)=F_{j}(x), \quad F=\bar{F}_{0}-\left(E_{1}+\cdots+E_{j-1}\right) .
$$

If (59) does not hold then we have (57) for $k=j-1$ and hence we have point (3). Thus we can assume (59).

$$
\left|E_{1}\right|>\left|\bar{F}_{0}\right|\left(\log \tilde{A}_{N_{1}}\right)^{-1}
$$

and hence 


$$
\left|\bar{F}_{0}-E_{1}\right|<\left|\bar{F}_{0}\right|\left(1-\left(\log A_{N_{1}}\right)^{-1}\right) \text {. }
$$

Also by Lemma 9 ,

$$
\left|E_{2}\right|>\left|\bar{F}_{0}-E_{1}\right|\left(\log \tilde{A}_{N_{2}}\right)^{-1}
$$

and hence

$$
\left|\bar{F}_{0}-\left(E_{1}+E_{2}\right)\right|<\left|\bar{F}_{0}\right|\left(1-\left(\log \tilde{A}_{N_{1}}\right)^{-1}\right)\left(1-\left(\log \tilde{A}_{N_{2}}\right)^{-1}\right) .
$$

It is clear that by induction

$$
\begin{aligned}
\mid \bar{F}_{0}-\left(E_{1}+\right. & \left.\cdots+E_{j}\right)|<| \bar{F}_{0} \mid \prod_{1}^{j}\left(1-\left(\log \tilde{A}_{N_{1}}\right)^{-1}\right) \\
& \leqq\left|\bar{F}_{0}\right| \prod_{1}^{j}\left(1-\frac{1}{2 i} \log C\right) \leqq\left|\bar{F}_{0}\right| \exp \left(-\frac{1}{2 \log C} \sum_{i=1}^{j} \frac{1}{i}\right) .
\end{aligned}
$$

Since $\sum_{i=1}^{j} 1 / i \rightarrow \infty,\left|\bar{F}_{0}\right| \exp \left(-(1 / 2 \log C) \sum_{1}^{j} 1 / i\right)$ is very small if $j$ is large enough. Thus for $k$ sufficiently large,

$$
\left|\bar{F}_{0}-\left(E_{1}+\cdots+E_{k}\right)\right|<\delta / 2 \text {. }
$$

We now attend to point (2). If

$$
\lambda=\left(2 A_{N_{j-1}}^{-2} \log \log A_{N_{j-1}}\right)^{1 / 2}, \quad \eta=.4,
$$

then for $C$ large enough,

$$
\int_{\bar{P}_{0}} \exp \left(\frac{\lambda S_{N_{j-1}}(x)}{f(x)}\right) d x \leqq \frac{3}{2}\left|\bar{F}_{0}\right| \exp \left(\frac{\lambda^{2} A_{N_{j-1}}^{2}}{2}(1+\eta)\right) .
$$

If

$$
\Gamma_{j}=\left\{x: S_{N_{j-1}}(x)>f(x) 2^{1 / 2}\left(2 A_{N j_{-1}}^{2} \log \log A_{N_{j-1}}\right)^{1 / 2}\right\}
$$

then from (60) it follows that

$$
\begin{aligned}
\left|\Gamma_{j}\right| & \leqq \frac{3}{2}\left|\bar{F}_{0}\right| \exp \left(\frac{\lambda^{2} A_{N_{j-1}}^{2}}{2}(1+\eta)-\lambda 2^{1 / 2}\left(2 A_{N_{j-1}}^{2} \log \log A_{N_{j-1}}\right)^{1 / 2}\right) \\
& \left.\leqq \frac{3}{2}\left|\bar{F}_{0}\right| \exp \left(\left(1+\eta^{-2}\right) 2^{1 / 2}\right) \log \log A_{N_{j-1}}\right) \\
& \leqq \frac{3}{2}\left|\bar{F}_{0}\right|(\log C)^{-1}(j-1)^{-1.4} .
\end{aligned}
$$

Clearly

$$
\left|\bigcup_{j=2}^{\infty} \Gamma_{j}\right| \leqq \frac{3}{2}\left|\bar{F}_{0}\right|(\log C)^{-1} \sum_{j=2}^{\infty}(j-1)^{-1.4}
$$


If $C$, and hence $\log C$ is large enough, this implies

$$
\left|\bigcup_{j=2}^{\infty} \Gamma_{j}\right|<\delta / 2
$$

Thus we have shown points (1), (2), and (3) and in consequence have completed the proof of the theorem.

\section{REFERENCES}

1. R. Salem and A. Zygmund, On lacunary trigonometric series, Proc. Nat. Acad. Sci. U.S.A. vol. 33 no. 11 (1947) pp. 333-338.

2. J. Ferrand and R. Fortet, Sur des suites arithmetiques equireparties, C. R. Acad. Sci. Paris vol. 224 (1947) pp. 516-518.

3. P. Erdös and I. S. Gal, On the law of the iterated logarithm, Nederl. Akad. Wetensch. Proc. Ser. A. vol. 58 (1955) pp. 65-84.

4. R. Salem and A. Zygmund, La loi du logarithme itêré pour les series trigonometriques lacunaires, Bull. Sci. Math. (2) vol. 74 (1950) pp. 209-224.

5. M. Weiss, On the law of the iterated logarithm for lacunary trigonometric series, Trans. Amer. Math. Soc. vol. 91 (1959) pp. 444-469.

6. G. Morgenthaler, A central limit theorem for uniformly bounded orthonormal systems, Trans. Amer. Math. Soc. vol. 79 no. 2 (1955) pp. 281-311.

University of Chicago,

Chicago, Illinois 